Seca Jean-Marie, 2011b, "Colère et enragement: la scène publique et le processus », Sociétés, n 114/4 (octobredécembre), pp. 195-106.

\title{
Colère et enragement : la scène publique et le processus
}

Jean-Marie Seca, 2L2S, Université de Lorraine

Résumé : Le but de cet article est de définir et analyser les grandes thématiques et les modalités d'approche de la colère dans les sociétés postmodernes. Deux concepts clés seront distingués (colère et enragement), décrivant la scénographie et le processus de ce phénomène multiformes. On indiquera qu'il y a deux autres modalités de scénarisation de la colère (l'une anomique et réactionnelle, l'autre de type divinatoire et démiurgique). On observe désormais une atomisation des « raisons de se fâcher » et de l'expérience colérique, tant dans les espaces publics sériels que dans ceux plus communautaires ou organisationnels. En fin d'article, on proposera de prendre en compte la position du leader dans le déclenchement d'effets d'intensification de l'enragement et de la colère.

Abstract: The purpose of this article is to define and to analyze important topics and modalities of approach of the anger in the postmodern societies. Two key concepts will be distinguished (anger and enragement), describing the multi-form scenography and the process of this phenomenon. We shall indicate that there are two other modalities of anger expression (the one on anomy state, and reactant, other one of the divinatory and demiurgic type). One observes henceforce an atomization of the "reasons for getting angry" and of the anger experiences, both in the serial public places and in those more community or organizational sides. At the end of article, we shall suggest considering the position of the leader in the release of effects of intensification of the enragement and the anger.

La colère et l'enragement sont des formes émotionnelles vécues tout autant individuellement (traits psychopathologiques ou héroïques) que collectivement (contagion, influences sociales, structures de retenue, de prêt et de canalisation). Ces états mentaux charrient des dimensions chaotiques, historiques et des valeurs contradictoires, évoquant l'inconscient, ses pulsions et ses conséquences déstructurantes, mais aussi l'intuition du présent, la vision du futur, l'anticipation d'événements, voire l'action mythique, si l'on se réfère à l'exemple fameux de la colère d'Achille, téléguidée par les dieux. L'ire divine elle-même et son évolution (mythologie du Jugement Dernier, Enfer) indique son ancrage archétypal et millénaire. Celle-ci se cristalliserait en une sorte de trésor biblique dans certains passages de l'Ancien Testament, d'après Peter Sloterdijk. Dans une première phase, la gestion de la colère de 
Dieu conduit à une mémoire légitimée de la haine, conservée notamment dans des Psaumes, sur les persécutions et rapports avec des ennemis du peuple d'Israël. Elle favoriserait aussi un rapport de purification et d'éducation établi par Dieu envers ses sujets (Sloterdijk, 2007). Tout indique, si l'on se base sur ces premiers exemples et sur bien d'autres, que l'on a à faire à un constituant déroutant, antique et multivalent des modalités d'influences sociales et culturelles intergroupes.

On peut donc souhaiter voir émerger un courant d'étude scientifique des colères postmodernes bien que de nombreux travaux sociologiques aient été consacrés aux émeutes urbaines (Mauger, 2006). Dans diverses circonstances, les états colériques se réduisent à la seule expression de la haine et à ses conséquences délétères ou aux phénomènes de la guerre, de l'extermination raciste ou terroriste. Certains mouvements de foules sont ouvertement et spontanément violents (pogroms, ratonnades, lynchages, vengeances, actes racistes). La colère les sous-tend et les dynamise. On ne s'occupera pas, dans cet article, de ces conduites quand elles se traduisent par ce que l'on veut éviter (la mort, la destruction d'autrui ou la discrimination ouverte d'un groupe). II faut évidemment questionner la colère dans ses effets indésirés et néfastes. C'est clairement l'un des objectifs essentiels, à moyen terme, de notre recherche. II n'est pas celui de cet article. Le contexte et l'envie de démocratie favorisent philosophiquement, l'émergence de mouvements de protestation mais ils ne permettent absolument pas de cautionner la violence ouverte et exterminatrice contre un groupe, des minorités ou des individus. Nous nous centrerons plutôt, dans cet écrit, sur la propagation des états colériques « acceptables » ou socialisées et sur leur sémantique contemporaine. Cette dissémination est patente car on assiste, de plus en plus, à une association des productions culturelles et artistiques à une tendance culturelle plus élargie de contestation de l'institué, se structurant à partir d'un imaginaire du ressentiment dont les supports nombreux sont sources d'imitations régulières et généralisées.

Dans un premier temps, nous nous occuperons de décrire ce que l'on pourrait appeler «la scénographie » (événement, contenus, nature des affects) et le « caractère processuel » de la colère, le but étant alors d'approcher son sens par deux versants (spatial et diachronique). On verra alors émerger des dimensions paradoxales, entre divination et esthétisation, effleurant les formes de violences destructrices sans y sombrer systématiquement. Ce caractère chaotique et structuré des colères se traduit par l'expérience de la transe moderne mais aussi dans des formes expérientielles fragmentées dont il s'agit d'interpréter la nature. La fragmentation des expériences colériques reflète-telle celle des formes de socialisation virtuelles ou plurielles contemporaines, tant dans ses dimensions politiques que sacrées? Le sentiment de honte associé à l'exhibition de sa propre rage est aussi présent que la revendication du stigmate ou de l'injustice pour lesquels on est censé se rebeller. 
Quoiqu'il en soit, il s'agit d'expériences à la limite de la déviance et de l'anomie, de la foule et de l'individualité minoritaire active. Le blâme, associé à la colère, est fortement déterminé par l'empreinte des idéologies dominatrices. II expose donc le porteur de colère à la répression ou au conformisme groupal et à l'archivage de ses rébellions. Nous conclurons cet article sur la question de l'aveuglement, non des masses mais des élites politiques et managériales.

\section{Scénographie et processus : approche sémantique de l'enragement}

Une source possible de la colère réside-t-elle dans un manque structural/structurant de type psychique et ontogénétique, comme on le présuppose pour l'explication naturaliste de la culture, dans le cadre de la théorie de la néoténie humaine (Dufour, 2005, 2007; Policar, 2010) ? Ce sentiment d'inaccomplissement et d'inachèvement constitutif peut-il être articulé à une frustration partagée socialement? Fait-il écho à un murmure archaïque sur le «tonnerre de Dieu» ou les violences fondatrices, transmis de génération en génération? Qu'il soit conservateur ou progressiste, un mouvement protestataire, politique, religieux ou esthétique commence toujours par une mobilisation vers l'impossible, autour d'une utopie radieuse et donc d'entités supra-ordonnées (ou de Grands Sujets, comme dirait Dany-Robert Dufour, op. cit.). Si l'on se base sur cette constante imaginaire et quasi magique des mouvements sociaux, la rage en constitue-t-elle le versant énergétique et moteur ? Ce type d'émotion peut-il devenir un objet d'étude sociologique ? Pourquoi les formes colériques seraientelles privilégiées alors que la palette expressive des «êtres non inhumains », pour reprendre une expression de Bernard Stiegler, est bien plus large (Stiegler, 2008) ? La colère, la furie, le ressentiment et la rage peuvent être inclus dans la catégorie générique « haine » qui serait l'un des sentiments fondamentaux, avec l'expérience du moi, l'amour, l'espoir, l'inquiétude, la tristesse et la joie (Maisonneuve, 1948).

Objets d'une anthropologie des émotions (Colletta et Tcherkassof, 2003; Rimé et Scherer, 1989 ; Rimé, 2005; Sartre, 1965), la colère et la furie représentent des formes socioculturelles quasiment universelles pour l'unique raison qu'elles sont notamment dérivées de réactions infantiles primordiales, de projection et d'introjection (M. Klein, A. Freud, Winnicott), se traduisant, en termes collectifs et sociétaux, en «délires de territorialité » schizoïdes ou paranoïdes (Deleuze et Guattari, 1972). Elles correspondent donc à une forme transculturelle maniaque, alternant avec l'abattement mélancolique. Comme les délectations moroses (Demeuldre, 2004), les esthétiques de la colère génèrent une imprégnation mentale bienfaisante et une imagination créatrice. Elles réexpriment probablement, sous une forme déguisée, rationalisée, des états intimes d'impuissance face à l'objet maternel, inévitablement regretté et recherché, et donc face à un monde extérieur plus ou moins menaçant. Elles 
Seca Jean-Marie, 2011b, "Colère et enragement: la scène publique et le processus », Sociétés, n 114/4 (octobredécembre), pp. 195-106.

consistent dans des expériences instables, socialisées et régressives, sur le plan du rapport à autrui. Leur universalité ne permet pas d'en définir la variété phénoménale. Mais elle explique peut-être l'aisance avec laquelle rage et colère sont «transvasées » et traduites en formes esthétiques et sociales hétérogènes.

La colère peut aussi être expliquée par la nécessité mécanique et pulsionnelle de réduire une dissonance cognitive dans un groupe ou chez un individu. Elle se déverserait, comme un acide, sur les faits sociaux insupportés. La psychanalyse appréhende ce phénomène de la même façon à partir d'une théorie de l'économie libidinale (trop-plein et déversement) et de la sublimation/expurgation. On peut aussi penser la rage comme une forme ascétique et travaillée, une sorte de représentation/scénarisation de l'action vengeresse ou de la reconnaissance de soi. Se profilerait, alors et dans le meilleur des cas, une pensée propre des minorités actives, fondée sur la consistance, une vision du monde, l'originalité et l'innovation, nécessitant une «dose de colère ou de rage » pour soutenir leur objectif de visibilité sociale. Ces conduites doivent, par conséquent, être évaluées de façon variée bien qu'elles offrent au regard de l'analyste une même surface de simplicité et de primarité. Elles peuvent conduire au pire comme au meilleur des comportements, être téléguidées ou spontanées, manipulées ou inspirées, aboutir ou non à des basculements moraux, sociétaux ou politiques ou à la violence pure. Les colères peuvent trouver leur « lit » expressif dans tout contexte, qu'il soit traditionnel, moderne ou postmoderne. Elles sont, par définition et dans l'approche que nous en proposons, des actes de communication sociale, politique et culturelle.

Ce continent expressif et sociologique est recouvert de paysages étranges et familiers. La colère est multivalente dans ses contenus et plurifactorielle par ses déterminations tant socio-économiques que culturelles, spirituelles, théologiques, sociologiques ou psychiques. Elle résulte de systèmes d'influences, d'apprentissages de ses modes d'expression et de représentations hétéroclites, tant pendant sa genèse que durant son plein développement pour ses victimes, ses agents, ses thésaurisateurs et les autorités répressives ou politiques. Elle interfère avec le champ d'étude des contagions sociales, vues, par Max Scheler, comme résultant d'interactions cumulatives d'excitations affectives et imitatives (Scheler, 2004). Elle circule et elle préexiste aux événements et aux phénomènes visibles de mécontentement dans ce qu'on peut appeler la « rage ». Elle serait donc à définir, si l'on acceptait cette nuance, comme la cristallisation et la scénographie de la rage. En tant que processus latent, la rage (et l'ennui) se gère dans le temps et pour des buts les plus inavouables au sein de diverses organisations publiques, privées et politiques. Elle est mentalisée et méditée par ses agents. Elle macère longuement et elle se diffuse parfois informellement sans qu'on sache discerner 
ses motifs et ses rationalisations. Elle est emmagasinée, congelée, réservée, retenue car la lâcheté, la prudence ou la peur empêchent les grands nombres d'exprimer régulièrement et ouvertement leur amertume. Elle se déclare, en tant que colère, avec force et bruit. Elle enflamme alors ses producteurs et ses consommateurs/spectateurs. Sous une forme explicite et publique, elle peut conduire à la structuration et à la rébellion temporaire d'un groupe ou carrément d'une société tout entière.

Scott Schieman confirme notre conception double (processus/scénographie) de ce phénomène. II distingue « ennui », «crise de colère » et « atténuation / chute de la colère » (Schieman, 2010). II y aurait donc des phases d'accès (et de sortie) aux autoroutes de la colère. Afin d'être plus précis, on dira que l'enragement peut être vu, en plus de l'ennui, comme un processus de montée vers la colère, analogue à celui l'état naissant de l'amour («innamoramento», en italien, signifiant «amour naissant »). L'enragement renverrait à la colère naissante, même s'il n'y a étrangement pas de mot pour dénommer l'« enragement » dans la langue de Dante. On distinguera donc le composé scénographique «colère » de son équivalent processuel «rage » (ou plutôt «enragement »), même si, conventionnellement et habituellement, les deux mots sont synonymes.

\section{Les conditions de l'exhibition colérique et la concentration divinatoire}

Une première remarque analytique peut faire avancer la réflexion: la rage n'est esthétisable, donc sublimée, que par son caractère non abouti et du fait d'une éthique expressive essentialiste. Dès qu'elle se « finalise », sa rutilance exhibitionniste et esthétique se mue en poussière relationnelle insensée. Le plus intéressant, dans son esthétisation, est son effet principal, c'est-à-dire son instrumentalisation, y compris par le colérique lui-même (et ses conseillers ès rage). II serait alors juste, bien vu et bon d'arborer les stigmates de la colère stylisée, même si la sagesse populaire et l'histoire des violences passées et récentes indiquent le contraire ou conseille la discrétion. Mais pour que cette culture colérique fasse son effet, il faut qu'elle se substantialise en une forme absolue et provocante, rejoignant alors la geste héroïque des personnages illustres de la mythologie ou de mythes plus contemporains. En dépit de cette instrumentalisation esthétique, en forme de stigmate ou de promesses de vengeances, nous vivons, depuis deux siècles en France, une époque paradoxale, à la fois expressive, essentialiste et spécifiquement bourgeoise et repliée. Cette exigence d'autolégitimation et d'autojustification, passant par l'excès et l'automortification, est considérée comme obscène dans les classes sociales supérieures et chez les snobs qui orientent les modes, le bon goût des salons mondains et les formes convenues de la conversation mesurée et caustique. Avec les cultures alternatives postdadaïstes et post-soixante-huitardes, le savoir-vivre prend d'autres formes et une stylisation spécifique. Les colères postmodernes pourraient donc découler en tant qu'effets seconds des 
Seca Jean-Marie, 2011b, "Colère et enragement: la scène publique et le processus », Sociétés, n 114/4 (octobredécembre), pp. 195-106.

valeurs-mêmes d'authenticité, d'expression émotionnelle du soi, de véracité des propos tenus, dénotant un engagement, d'une expérimentation narcissique de la communication et de la fusion de soi avec le groupe qui reçoit, par compensation, toutes les vertus de la thérapie qu'il est impossible d'activer seul. Ce faux nouveau savoir-vivre groupal et cool, dit « sans tabou » ou « expressif », est erratique dans ses codes d'adoption et ses rituels. Les formes de son déploiement dépendent des subjectivités en coaction. II se ritualise autour de feelings instantanés et de sympathies partagées et montrées. Cependant, il participe d'une éthique de l'excès émotionnel. L'esthétisation de la rage est donc aussi, dans le même mouvement, un moyen de faire passer cette éthique et ses systèmes représentationnels favorisant un mode d'être, dit « essentialiste ».

Deuxième remarque analytique: il y aurait des colères réactionnelles, contre-dépendantes, disséminées, et d'autres plus divinatoires. Le processus d'enragement et sa scénographique colérique sont accompagnés d'une activité représentationnelle tout en voisinant avec un état régressif et pathologique. Une accentuation de la concentration sur les sensations internes des individus se déroule durant l'ascension passionnelle colérique. Par contrecoup, un excès dans la perception des objets (au sens général de ce terme) du monde modifie la conscience du sujet. Une lumière plus crue est projetée sur les objets ou les personnes convoitées ou enviées par le déçu de soi et de son environnement relationnel qu'est l'enragé. Une montée en puissance de cette perception est alors alliée à un besoin de sortie énergétique et d'emprise magique sur le monde. Le chamane représente un prototype professionnel et rituel de ce phénomène d'excès, accompagné de spiritualité surnaturelle et divinatoire et d'un mixte d'intellectualisation, de furie, de transe et d'émotion brute (Hell, 1999, 176-177). Chez les Bouriates de Sibérie orientale, la rage est qualifiée par le terme «galzuu ». On y observe l'existence d'une véritable furie chamanique, nettement différenciables de conduites pathologiques. Le caractère pacifiant du rituel et de la fonction de chamane est d'ailleurs opposé à une sorte de démesure typiquement anomique de l'homme ordinaire.

«Ce terme de galzuu est couramment traduit par "endiablé, enragé, fou". II s'applique aussi bien au chien malade de la rage, acharné à mordre, qu'à l'homme ordinaire, rendu, "par les esprits", agressif à l'égard de son entourage, et qu'à l'état auquel le chamane doit absolument parvenir au cours du rituel. À la différence du chamane, l'homme ordinaire devenu galzuu est tenu pour malade : on le dit atteint par une rage proche de celle du chien et autres animaux susceptibles d'en être frappés. Ce concept de galzuu est largement utilisé dans le discours chamanique et figure, à ce titre, dans le Dictionnaire des termes chamaniques de Manžigeev : l'état de chamane qu'il désigne est, dit cet auteur, le point culminant de la séance, indispensable à son succès [...]. 
L'expression galzuuran böölexe, qui signifie littéralement "chamaniser frénétiquement", correspond à l'entrée en extase [...], c'est-à-dire en contact avec les esprits. Manžigeev [...] présentant l'expression galzuu böö, "chamane enragé", comme désignant le chamane en état de furie violente et bestiale, au moment culminant de sa séance ou "extase" [sic], ajoute que cet état est souvent le fruit d'une simulation, destinée à concrétiser le contact avec les esprits. II faut comprendre que le chamane remplit son rôle de façon plus ou moins convaincante » (Hamayon, 1990, p. 522.)

L'état enragé n'est donc pas toujours considéré comme un pathos politiquement incorrect tout en ressortissant néanmoins d'une sociologie de la santé mentale. On peut utiliser à son égard une catégorisation psychiatrique ou bien l'envisager comme une expérience mentale puissante. II se définit par un abandon dans l'immanence quotidienne, mêlé à une conscience accentuée de l'environnement et à la nécessité, pour tout groupe, de construire des rituels d'insertion et de provocation sociétale. L'ennui semble inséparable, comme phase de ressenti de l'ambivalence, de l'émergence colérique et de l'invention d'un possible. Roger Bastide relève que l'une des quatre fonctions des rites de possession africains est la divination ${ }^{1}$ (Bastide, 1972, pp. 91-93). Outre son origine mystique, son caractère théâtral et ambivalent, générée différemment selon le statut de celui qui l'active, la rage et ses dérivations voisinent avec l'état de transe, sans y être complètement assimilable. La divination des enragés est, quant à elle, soit une sorte d'anticipation très mentalisée et collective des modifications sociétales nécessaires dans un espace géopolitiques donné, soit une sorte de self-fulfilling prophecy, créant progressivement la réalité hallucinée dans les manifestations colériques. L'exemple de la révolution iranienne illustre bien l'effet de «création » normative, institutionnelle et politique de colère religieuse et nationale orientée.

\section{La fragmentation des rages et la peur du blâme}

La colère fait parfois suite à de la frustration, engendrée par l'issue défavorable ou inéluctable d'un conflit, d'une opposition, d'une convoitise ou d'un espoir contrarié. Elle frôle alors le poison du ressentiment (Angenot, 1996; Scheler, 1970 ; Nietzsche, 1887). Cela rend l'appréhension de ce phénomène encore plus difficile. On peut envisager une excroissance virtuelle, amère, de cet état mental complexe, mêlé et «mêleux² », donnant lieu à l'instauration de structures idéologiques et

\footnotetext{
1 Voir d'ailleurs pour plus de détails sur l'art divinatoire et ses rapports avec la transe : Dufoulon, 1997.

${ }^{2}$ Cet argot bas normand désigne les conduites de curiosité voyeuristes et conflictuelles dans les espaces compartimentés et convoités du bocage où Jeanne Favret-Saada avait enquêté sur la sorcellerie.
} 
politiques colinéaires, impliquant ce que Sloterdijk qualifie de « points de collectes de la colère opérant sur de vastes espaces et coordonnés par une mise en scène visionnaire ». L'administration de la colère est opérée par des idéologues froids et calculateurs capables de penser des slogans idéalistes évoquant l'amertume autant que la fierté Ces «banquiers de la colère», professionnels de la canalisation de cette « lave enragée » et du ressentiment œuvrent alors obstinément (Sloterdijk, op. cit., pp., 90-99).

La rage, dans sa forme postmoderne, est cependant éminemment fragmentée. Le capitalisme contemporain facilite ce que Sloterdijk appelle le « retardement de l'apoplexie » associé, selon lui, à l'érotisation de l'éthique quotidienne (ibid.). Par des réseaux de dissidence éparpillée internationalement, elle se propage de façon corpusculaire. Elle émerge, par bouquets épars, de l'expérience-même de la globalisation culturelle. Elle s'instille dans les systèmes sociotechniques et administratifs des organisations publiques, privées et politiques. Elle s'empare des masses sérielles comme des bandes en dérive ou des électorats indécis. La conduite enragée est, alors et à la fois, une erreur de comportement et une sorte de réaction lucide et structurée. Elle fait partie intégrante des actions de masse tout en n'étant repérée nulle part (Ortega Y Gasset, 1937). Elle épouse l'évolution des formes urbaines et des modifications géopolitiques ou démographiques. Elle coïncide avec l'impact croissant et psychosociologique des grands nombres. II est difficile de mettre en place des observations empiriques calibrées et reproductibles de ce phénomène fragmenté. Dans quels champs ou terrains peut-on l'analyser ? Diverses possibilités s'offrent au regard.

L'évolution des mouvements sociaux vers des préoccupations identitaires depuis une quarantaine d'années peut offrir, des terrains d'observation sur la variété des protestations centrées sur le soi « tribalisé » (Maffesoli, 1988). II s'agit d'enquêter non pas uniquement sur la motivation et l'objectif de revendications, mais sur les formes d'émergence et les états émotionnels mis en scène et ressentis. Par ailleurs, les modes de management contemporains vantent une plus forte flexibilité de l'emploi et des relations sociales et un pilotage des engagements professionnels par les compétences et par objectifs. Ce mode financier et flexible d'administration des organisations est organisé autour d'un marché opaque et imprévisible, de salaires individualisés et précaires et de dissymétries entre des secteurs protégés et des strates exposées à l'insécurité d'emploi. II en résulte notamment une augmentation des suicides et des souffrances au travail (Desjours, 2009 ; Menger, 2002), des plaintes de harcèlement moral et d'abus d'autorité. Le resserrement égalitariste des pyramides hiérarchiques est accompagné d'une trop forte valorisation du groupe d'égaux professionnels, comme dépositaires d'une légitimité illusoire (Sennett, 2000). Là aussi, l'état de rage circule et se formule de différentes façons. 
La diversité et la fragmentation des états de rage contemporains s'accentuent avec l'emprise des représentations égotistes du bien commun et de soi, générées lentement hors des organisations et en leur sein. Elles s'intensifient aussi en fonction de conditionnements et cultures mécaniques de masse organisées autour de sollicitations émotionnelles engendrant dépendance et asymétrie. Les industries de programme diffusent une culture du ressentir par effets de contagion et d'amplification, d'identification et d'accroissement des émotions programmées, construites. Dans un tel cadre, la valorisation et l'exhibition de la sympathie, au sens large de ce mot, dans les rapports interindividuels généreraient des effets paradoxaux. II pourrait bien en résulter une forme plus courante d'égotisme, de vécu de déclassement social, de comparaison de soi avec autrui et d'idéalisation frustrante, marquée par des modèles de différenciation sociale. Pour François Dubet, les états de rage résulteraient d'un effet de domination subie et sans visage, de la fascination pour la force et du nihilisme (Dubet, 1987, pp. 90-92). Malgré sa fragmentation croissante, il serait possible de contrôler la colère (et les enragés) puisque ces états mentaux renforcent les structures de gouvernement autocratiques en impliquant une régression «rébellion/soumission ». Les émeutes sont bien plus les fruits empoisonnés d'un aveuglement et d'une obstination des pouvoirs que d'une incapacité technique à contrôler leur apparition et leur atténuation. Certains incidents en augmentation ou endémiques indiquent néanmoins que les états de rage sont permanents et affleurants. On pensera au nombre journalier et récurrent d'incendies criminels (en forêt ou bien dans les centres urbains), en France ; aux agressions en milieu scolaire ; ou à la hausse du nombre de profanations 3 .

Ces états mentaux recèlent des parcelles de tonicité malgré leur violence brute et leur morbidité marginale. Ils parcourent les styles et contenus musicaux rock. Ils marquent nombre d'événements, de révoltes et de révolutions, des enragés de 1792 aux Damnés de la Terre communards (Furet, 1988 ; Miquel, 1988 ; Régaldo, 1987 ; Rose, 1965). L'affrontement du malheur et de la pauvreté nécessite aussi que l'état de rage soit entretenu (Declerck, 2001 ; Seca, 2010). La colère avilit cependant celui qui s'y adonne et l'expose à la réprobation. C'est pourquoi il connote la personne qui s'y livre. La violence morale au travail est souvent accompagnée de honte, d'évitement, de brutalité revancharde des victimes ou des persécuteurs et de difficultés juridictionnelles. II faut prendre au sérieux cet effet d'humiliation. Le choix du comportement colérique requiert donc un sens aigu du calcul ou, au contraire, une culture de l'abaissement de soi. Dans l'adoption d'un code d'enragement, on s'isole tout en mobilisant des affects collectifs groupaux et de masse. On diminue inévitablement ses aptitudes à la réflexivité. On fait le choix des affects et des modes d'expression infralangagiers et d'une sorte de

\footnotetext{
3 « Les profanations sont en hausse constante », Article paru dans l'édition du Monde du 2 novembre 2010 : « En huit mois,
} 387 dégradations visant des lieux de culte catholiques, juifs ou musulmans et des cimetières ont été recensées ». 
schème cognitif, assimilable à une « intime conviction ». Cette peur du blâme maintient les citoyens dans une retenue enragée, un ennui. Elle conduit certains d'entre eux à de la réticence dans l'engagement de protestation (qu'il s'agisse de grèves ou de mouvements sociaux plus globaux ou identitaires).

\section{L' « innocence » infernale des gouvernants}

L'émergence colérique peut advenir désormais dans tout espace sociétal, au moment le moins attendu. La prodigieuse révolte tunisienne et ce qu'il est convenu d'appeler « Le Printemps Arabe », de janviermai 2011, l'illustrent parfaitement. Les gouvernants et les élites diplomatiques et techno-économiques hexagonales ne pouvaient pas imaginer la chute de Zine el-Abidine Ben Ali. Cet aveuglement est assez typique de la position de domination, « sûre de soi ». L'épisode ridicule du Rubygate en Italie le montre bien : son impression fragile d'invulnérabilité n'est qu'un nuage toxicomaniaque autour de la figure clownesque et piteuse du Cavaliere. Pour ce qui concerne la France, l'héritage du moule gouvernemental de l'Ancien Régime français et du monarchisme se détecte, depuis le début du 19 e siècle, dans les restaurations et les réactions diverses qui le jalonnent. II est actif dans l'instauration de la Ve République, à partir de 1958-1962. On le voit aussi à l'œuvre dans l'orientation managériale hyper-rationaliste de nombreux "grands patrons », énarques et polytechniciens. Les mouvements protestataires, tant syndicaux qu'identitaires, n'en deviennent alors que plus véhéments, en contreréaction à cet aveuglement teinté de suffisance.

Cette forme dominatrice des négociations prévaut dans les espaces organisationnels. Elle s'incarne dans la volonté d'instaurer une dissymétrie dialogique, en mettant en avant une décision prise « rationnellement » puis son « explication » aux masses errantes des « cavernes » postmodernes. Les excès colériques trouvent là leur lit naturel et des sentiments fondés de persécution ou de mépris. Le déclenchement d'émeutes se déroule souvent avec les meilleures intentions des autorités (Zinoviev, 1978). Peu à peu, et parfois très vite, les malentendus sont générés, les abus naissent et renaissent, par l'exercice naturel du pouvoir et de ses vertiges. La jubilation éprouvée durant l'accès au contrôle d'autrui n'a jamais été étudiée avec sérieux. Le pouvoir a toujours été analysé du point de vue des effets sur la personne à conduire et sur les techniques de persuasion (Deschamps et Beauvois, 1996, pp. 22-133). C'est dommage que l'on n'ait pas suffisamment observé cet état mental, entre pathologie et philosophie de l'existence, vécu aux postes de commandement. La fascination du pouvoir et sa magnétisation sont fascinantes à étudier. Au lieu de supposer le leader uniquement paré de qualités impressionnantes (actifs et innovants), on devrait vérifier si la situation de domination engendre ou non un rapport trouble à l'espace social, avec, d'un côté, certaines élites déportées vers le point aveugle de 
la fascination, et, de l'autre, des groupements enragés, segmentés, plus ou moins actifs, envieux, jaloux, vindicatifs, politisés ou artistiques.

\section{Conclusion}

On doit souhaiter le développement d'un champ de recherche structuré, équivalent à ce qui se fait aux États-Unis (Potegal et al., 2010). Des enquêtes organisationnelles sur les ambiances de travail ou le savoir-vivre en ayant comme objets focaux d'observation les emportements, l'irrespect, l'enragement, l'aveuglement lié au statut de domination et les colères pourraient être réalisées. Divers outils triangulés et multiréférentiels de mesures, articulés aux principes d'anthropologie des rituels (Maisonneuve, 1999 ; Picard, 1983) à des indicateurs construits et connus (questionnaires et échelles sur les représentations et attitudes durant ou après les colères ou les conflits notamment: Deffenbacher, 2002 ; Ost 2004 ; Tjosvold et Su, 2007) peuvent servir à ce but. Une enquête plus qualitative sur la définition et la mémoire colérique chez des individus de différents niveaux de certification éducative et professionnelle devrait compléter ce dispositif d'observation. Ce serait un premier mouvement de cette série de travaux, en complément à une appréhension théorique et socio-historique de l'objet. L'autre objectif à atteindre pour parvenir à une socio-anthropologie de ce phénomène est de globaliser l'étude des colères en les rapportant à une écologie urbaine qui se généralise (des réseaux internet aux zones rurbaines en passant par les centres denses des grandes villes) et aux phénomènes de mondialisation. II ne s'agit pas seulement d'étudier les émeutes quand elles se produisent, en tant que « spectacle », ni de voir émerger des mouvements sociaux renversants. Le but de cette approche des colères urbaines est d'en filer la quotidienneté quitte à en faire, comme beaucoup de chercheurs, une obsession.

\section{Bibliographie}

Angenot Marc, 1996, Les Idéologies du ressentiment : essai, Montréal, XYZ.

Bastide Roger, 1972, Le Rêve, la transe et la folie, Paris, Flammarion.

Colletta Jean-Marc et Tcherkassof Anna (éd.), 2003, Les Émotions: cognitions, langage et développement, Sprimont, Mardaga.

Declerck Patrick, 2001, Les Naufragés. Avec les clochards de Paris, Paris, Plon.

Deffenbacher Jerry, Oetting Eugene et DiGiuseppe Raymond, 2002, «Principles of Empirically Supported. Intervention applied to Anger Management », The Counseling Psychologist, Vol. 30, $\mathrm{n}^{\circ}$ 2, pp. 262-280.

Dejours Christophe, 2009, Suicide et travail. Que faire ?, Paris, PUF. 
Seca Jean-Marie, 2011b, «Colère et enragement: la scène publique et le processus », Sociétés, n 114/4 (octobredécembre), pp. 195-106.

Deleuze Gilles et Guattari Félix, 1972, L'Anti-CEdipe. Capitalisme et schizophrénie, Paris, Minuit.

Demeuldre Michel (Éd.), 2004, Sentiments doux amers dans les musiques du monde. Délectations moroses dans le blues, fado, tango, flamenco, rebetiko, p'ansori, ghazal..., Paris, L'Harmattan.

Deschamps Jean-Claude et Beauvois Jean-Léon, 1996, Des attitudes aux représentations. Sur la construction sociale de la réalité, Grenoble, PUG.

Dubet François, 1987, La Galère : jeunes en survie, Paris, Fayard.

Dufour Dany-Robert, 2005, On achève bien les hommes. De quelques conséquences actuelles et futures de la mort de Dieu, Paris, Denoël.

Dufour Dany-Robert, 2007, Le Divin Marché. La révolution culturelle libérale, Paris, Denoël.

Furet François, 1988, La Révolution (deux tomes), Paris, Hachette.

Hamayon Roberte, 1990, La Chasse à l'âme. Esquisse d'une théorie du chamanisme sibérien, Nanterre, Société d'ethnologie.

Hell Betrand, 1999, Possession et chamanisme. Les maîtres du désordre, Paris, Flammarion.

Maffesoli Michel, 1988, Le Temps des tribus. Le déclin de l'individualisme dans les sociétés de masse, Paris, Klincksieck.

Maisonneuve Jean, 1999, Les Conduites rituelles, Paris, PUF (3e éd. corrigée).

Maisonneuve Jean, 1948, Les Sentiments, Paris, PUF.

Mauger Gérard, 2006, Les émeutes de novembre 2005 : une révolte politique, Paris, Le Croquant.

Menger Pierre-Michel, 2002, Portrait de l'artiste en travailleur. Métamorphoses du capitalisme, Paris, La République des Idées / Le Seuil.

Miquel Pierre, 1988, La Grande révolution, Paris, Plon.

Ortega Y Gasset José, 1937, La Révolte des masses, trad. fr., Paris, Stock (1re éd. en langue castillane : 1930).

Ost David, 2004, «Politics as the Mobilization of Anger. Emotion in Movement and in Power », European Journal of Social Theory, vol. 7, $n^{\circ}$ 2, pp. 229-244.

Picard Dominique, 1983, Du Code au désir. Le corps dans la relation sociale, Paris, Dunod.

Policar Alain, 2010, «Néoténie, croyance et naturalisme », Les Cahiers de Psychologie politique, numéro 17, juillet, URL: http://lodel.irevues.inist.fr/cahierspsychologiepolitique/index.php?id=1687.

Potegal Michael, Stemmler Gerhard et Spielberger Charles (éd.), 2010, International Handbook of Anger. Constituent and Concomitant Biological, Psychological, and Social Processes, New York, Springer. 
Régaldo Marc, 1987, « Le radicalisme révolutionnaire », in Pascal Ory (éd.), Nouvelle histoire des idées politiques, Paris, Hachette, pp. 152-165.

Rimé Bernard et Scherer Klaus (éd.), 1989, Les Émotions, Neuchatel, Delachaux et Niestlé.

Rimé Bernard, 2005, Le partage social des émotions, Paris, PUF.

Rose Reginald, 1965, The "Enragés": Socialists of the French Revolution, Victoria, Melbourne University Press.

Sartre Jean-Paul, 1965, Esquisse d'une théorie des émotions, Paris, Hermann (1're édition : 1938).

Scheler Max, 2003, Nature et forme de la sympathie. Contribution à l'étude des lois de la vie affective, Paris, Payot (1re éd. en langue allemande : 1913).

Scheler Max, 1970, L'Homme du ressentiment, trad. fr., Paris, Gallimard (1re éd. en langue allemande : 1912).

Schieman Scott, 2010, «The Sociological Study of Anger: Basic Social Patterns and Contexts », in Michael Potegal et al. (éd.), 2010, International Handbook of Anger. Constituent and Concomitant Biological,Psychological, and Social Processes, New York, Springer, pp. 329-347.

Seca Jean-Marie, 2010, Conduites minoritaires et représentations sociales. Entre dynamiques culturelles et tendances anomiques, Sarrebruck, Éditions Universitaires.

Sennett Richard, 2000, Le Travail sans qualité. Les conséquences humaines de la flexibilité, trad. fr., Paris, 10/18/Albin Michel (1re édition en langue anglaise : 1998).

Sloterdijk Peter, 2007, Colère et temps. Essai politico-psychologique, Paris, Libella Maren Sell (1 re édition en langue allemande : 2006).

Stiegler Bernard, 2008, Prendre soin (tome 1) : de la jeunesse et des générations, Paris, Flammarion. Tjosvold Dean et Su Fang, 2007, « Managing Anger and Annoyance in Organizations in China. The Role of Constructive Controversy », Group \& Organizations, vol. 32, n 3 (juin), pp. 260-289. Zinoviev Alexandre, 1978, L'Avenir radieux, Lausanne, l'Âge d'homme. 